\title{
A moving window approximate entropy in wavelet framework for automatic detection of the onset of epileptic seizures.
}

\author{
N. Arunkumar ${ }^{1 *}$, K. Ramkumar', V. Venkataraman ${ }^{2}$ \\ ${ }^{1}$ Department of Electronics and Instrumentation Engineering, Sastra University, Thanjavur, India \\ ${ }^{2}$ Department of Mathematics, Sastra University Thanjavur, India
}

\begin{abstract}
Epilepsy is, in general, a diseased condition where the brain fires abnormal signals, which results in convolutions in the muscles-which occurs suddenly to the patients. In this work, we prescribe a novel method to automatically identify the onset of epileptic seizures. A moving window approximate entropy (ApEn) is run over the Electroencephalogram (EEG) signal with the epileptic seizures. ApEn value drops at the conjuncture of the onset of the epileptic seizures. This ApEn characteristic drop is considered as feature for detecting the onset. Moreover this ApEn characteristic drop is enhanced using wavelet transform forming the feature to be in ApEn wavelet framework. Three neural networks namely Feed forward Back propagation, Elman and Radial basis networks is employed for automatically detecting the onset of the epileptic seizure. They are compared with each other for their performance with ApEn as feature and ApEn in wavelet framework as feature. It was found that radial basis network is giving a better overall accuracy of $94 \%$, when their inputs are features being ApEn in wavelet framework. Additionally, the onset sample detected by the algorithm and manually identified onset sample by the encephalographers are compared and it was found that our algorithm was able to detect the onset of the epileptic seizures with an average detection delay of only 0.2 second for the 200 segments of EEG considered across 31 patients, which is low compared to earlier works in the literature.
\end{abstract}

Keywords: Approximate Entropy (ApEn), Epileptic seizure, Electroencephalogram (EEG), Spike.

Accepted on January 17, 2017

\section{Introduction}

Electroencephalogram (EEG) is discovered in 1930 [1]. It is a recording of the activity of the brain. It was also able to record the abnormal patterns arising in the brain. These abnormal patterns are called as epileptic seizures. About 50 million people i.e. roughly $1 \%$ of population in this world have epilepsy [2,3]. A person having this disorder is not easily received by common people even to the degree of prohibiting marriages. A person with this disorder experiences sudden seizures resulting in convolutions of his muscles and experiences a strange behaviour. Although by the disease itself does not lead to dangerous condition, rather it can be dangerous if the person is swimming or driving, as it can lead to loss of consciousness or disturbances in the movements or disturbances in the mental state. Seizures are of different types. They are classified primarily based on the source of the seizure within the brain namely localized or distributed. In the localized seizures are named as partial or focal seizures and distributed or nameless generally seizures. Partial seizures are further classified as simple partial seizure and complex partial seizure. If ones awareness is unaffected then it is called as simple partial seizure and if ones awareness is affected it is called as complex partial seizure. And generally seizures are classified according to the effect on the body but all of them involve loss of consciousness. These include absence (petit mal), myoclonic, clonic, tonic, tonic-clonic (grand mal), and atonic seizures. In general they have ictal period where there are seizures and a period preceding that called pre ictal period. Epileptic seizures can be recorded along with recording of the Electroencephalogram (EEG).

A lot of works are done in the automatic detection of epileptic seizures. Broadly speaking, researchers have used long term EEG data or EEG data segments for detecting the epileptic seizures. Bonn University database has EEG segments of a fixed duration of three different categories namely normal, ictal and inter ictal. Many researchers have employed different algorithms with this database and classified the normal, ictal and inter ictal EEG and reported accuracies as high as 99\% [4]. In terms of techniques, starting from the 1970's different methods has been employed. Initially heuristic and descriptive methods were used for the detection of epileptic seizures [5]. Later time domain methods, frequency domain methods, time frequency domain methods and other non linear methods were all attempted for seizure detection [6]. Linear discriminant analysis (LDA), histogram methods were also used for automatically detecting the epileptic seizures [7,8]. Seizure 
termination was identified using sample entropy [9] Differential operation was also used to identify the seizures [10]. Recurrence quantification Analysis (RQA) [11], Higher order spectra (HOS) [12], Hurst exponent (H) [13.], different entropies [14] were all employed for the identification of epileptic seizures. There are also works where they have employed wavelet transforms with single level analysis for detecting the epileptic seizures [15,16]. Multilevel wavelet approach was also employed by Indiradevi et al. for automatically detect the epileptic spikes [1]. Sharma et al. has used Fast Fourier transform (FFT) for extracting features from EEG as a first step and employed these features to the neural network for identifying the seizure [17].

Works are also done for automatically detecting the onset of the epileptic seizures. Different methods of machine learning algorithms with pattern recognition such as neural networks [18-24], support vector machines [25,26], KNN classifier [27], Bayesian classifier [28] have been implemented. Features such as correlation dimension [29], correlation density [30], Lyapunov exponent [31], Kolmogorov entropy [32] have been used for onset detection. Fourier transform was also implemented for onset detection of epileptic seizures [33-35]. Some researchers have also used wavelet transforms [36-38] to detect the seizure onsets. Details on the techniques and results of the different studies are discussed and compared with our technique in the discussion section. In our work, we are proposing a novel technique involving ApEn characteristic drop enhanced by wavelet transform which forms the feature: ApEn in wavelet framework to identify the onset of epilepsy which no one has yet used as for the knowledge of the author for onset detection of epileptic seizures. We achieved an average detection delay of only 52 samples from the onset of epileptic seizure, which comes out to be 0.2 seconds for 256 samples per second sampling rate of acquisition of the EEG signals, with an overall accuracy of $94 \%$ across the 200 data considered from 31 patients.

The paper is organized as follows, section 2 presents the description of the data which we have used in this work, introduces ApEn, moving window ApEn, wavelet transform which enhances the characteristic drop of the ApEn at the conjuncture of the seizure, different neural networks which are employed for automatic onset detection and the performance measures to evaluate the accuracy of the onset detection of the neural networks. Section 3 presents the results obtained in this work. Salient review of different techniques related to automatic epileptic seizure detection is discussed along with comparison of the results in the section 4 . The conclusion and future scope is given in section 5 .

\section{Methods and Materials}

In this section, we describe the methods that we have employed for automatic detection of the onset of epileptic spikes.

\section{Data}

The EEG data for this work was collected from the Department of Neuromedicine, Thanjavur Medical College Hospital, Thanjavur. The dataset contains two types, i.e., normal EEG, normal EEG followed by the onset of epileptic seizures. The EEG was recorded using a neuroscan machine Neuromax from Medicaid systems with 64 channels, $256 \mathrm{~Hz}$ sampling rate and a 16 bit A/D converter. Two encephalographers were asked to extract a standard length of artefact free EEG data - to avoid subjective errors. The standard length is formed in the following way: 16 seconds before the onset of epileptic seizures and 16 seconds after the onset of epileptic seizures is formed as one segment of data with the total duration of 32 seconds. A total of 200 such segments of EEG data are extracted from the 31 patients involved in the study.

\section{Approximate entropy (ApEn)}

ApEn is a recent measure to find the regularity of a signal. This algorithm was proposed by Pincus [39].

\section{ApEn calculation}

ApEn is a statistic for measuring regularity that quantifies the unpredictability of variations in a time series, $\mathrm{T}$ (i). The presence of regular patterns of variations in a time series makes it predictable. A time series which has many similar patterns has a lower ApEn relatively and a time series with random patterns has relatively larger ApEn. The algorithm for calculating ApEn has been published in different articles [40-43]. A general short summary of the calculations for ApEn is given as below.

Consider a sequence QM, which consists of $\mathrm{M}$ continuous measurements $\mathrm{T}$ (1), $\mathrm{T}$ (2), ... , T (M). To compute approximate entropy for this sequence, values have to be chosen for the parameters $m$ and $r$ in the function ApEn (QM, $\mathrm{m}, \mathrm{r}$ ). The parameter $\mathrm{m}$ represents the pattern length and $\mathrm{r}$ is the criteria of similarity. $P_{m}$ (i) is a vector of a subsequence of $\mathrm{m}$ measurements starting at measurement $\mathrm{i}$ within the sequence QM. Two patterns are considered as similar if their difference between any of the measurements in the patterns is less than $r$. Now let us consider the set Pm of all patterns of length $\mathrm{m}$ within QM. We may now define $\mathrm{C}_{\mathrm{im}}(\mathrm{r})=\mathrm{n}_{\mathrm{im}} /(\mathrm{N}-\mathrm{m}+1)$, where $\mathrm{n}_{\mathrm{im}}(\mathrm{r})$ is the number of patterns in Pm that are similar to $\mathrm{P}_{\mathrm{m}}(\mathrm{i})$ (given the similarity criterion $r$ ). The quantity $C_{i m}(r)$ is the fraction of patterns of length $m$ that resemble the pattern of the same length that begins at interval $i$. We can calculate $C_{i m}(r)$ for each pattern in $\mathrm{P}_{\mathrm{m}}$, and we define $\mathrm{C}_{\mathrm{m}}(\mathrm{r})$ as the mean of these $C_{i m}(r)$ values. The quantity $C_{m}(r)$ expresses the prevalence of repetitive patterns of length $\mathrm{m}$ in SN. Finally, we define the approximate entropy of $\mathrm{SN}$, for patterns of length $\mathrm{m}$ and similarity criterion $r$, as $\operatorname{ApEn}\left(\mathrm{S}_{\mathrm{N}}, \mathrm{m}, \mathrm{r}\right)=\ln \left[\mathrm{C}_{\mathrm{m}}(\mathrm{r}) / \mathrm{C}_{\mathrm{m}}\right.$ $\left.{ }_{+1}(\mathrm{r})\right]$ i.e., as the natural logarithm of the relative prevalence of repetitive patterns of length $m$ compared with those of length $\mathrm{m}+1$.

Thus, if it is found that similar patterns in a time series, ApEn estimates the logarithmic likelihood that the next intervals after 
each of the patterns will differ (i.e., that the similarity of the patterns is mere coincidence and lacks predictive value). Smaller values of ApEn imply a greater likelihood that similar patterns of measurements will be followed by additional similar measurements. If the time series is highly irregular, the occurrence of similar patterns will not be predictive for the following measurements, and ApEn will be relatively higher.

It should be noted that ApEn has significant limitations, notably its strong dependence on sequence length and its poor self-consistency (i.e., the observation that ApEn for one data set is larger than ApEn for another for a given choice of $m$ and $r$ should, but does not, hold true for other choices of $m$ and $r$ ). Hence in this work, we have kept a standard number of samples for the calculation of ApEn.

\section{ApEn for a standard Periodic and Random signal}

We have generated three standard signals. Firstly a sine wave, secondly a square wave and thirdly a sawtooth wave each with a frequency of $1 \mathrm{KHz}$. The ApEn code is written in Matlab 7 and it is calculated for each of these standard signals. It is found that the ApEn values for these signals are 0.0026, 0.0010 and 0.00002 respectively. The three signals and their corresponding ApEn values are shown in the Figure 1. It is inferred from this that for a periodic signal, the ApEn value is almost zero. A random signal is also generated and ApEn is calculated for the same and it was found to be 0.8358 . The random signal and its corresponding ApEn value are shown in Figure 2. From this it is inferred that ApEn value will be higher for a random signal than a periodic signal.

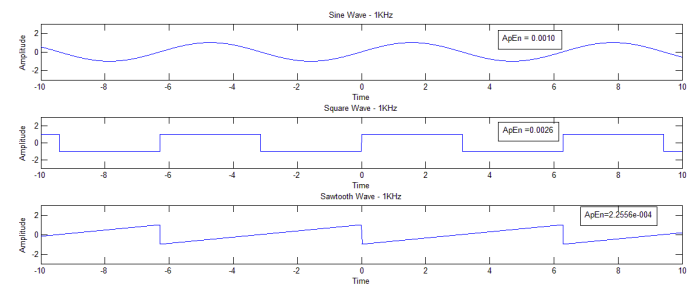

Figure 1. ApEn Values for a Standard Periodic signals.

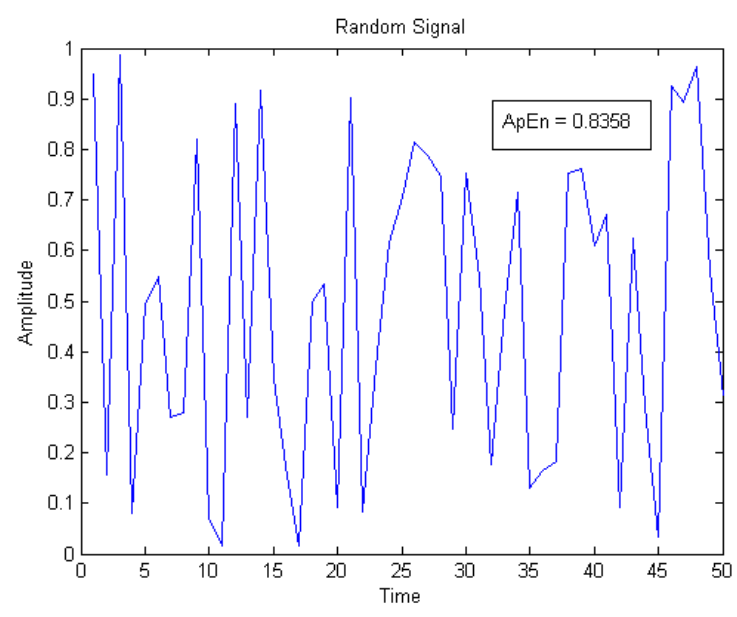

Figure 2. ApEn value for a Random Signal.

\section{ApEn for a normal EEG and an Epileptic EEG}

A normal EEG signal is almost like a random signal. There is no periodicity in a normal EEG signal. On the other hand, the doctors say that an Epileptic EEG is very less random and has some form of repetitive spikes or spike and wave structure. Therefore the ApEn value for a normal EEG and an Epileptic EEG must definitively be different. Hence this feature can be used as a feature for detecting the onset of Epileptic EEG. This is described in the following section.

\section{Moving window ApEn}

At the first step, the length of an epileptic spike in terms of number of samples for a spike is determined. For this 20 epileptic spikes from different patients at different timings were located with the help of two encephalographers to avoid subjective errors. From this, the average number of samples for the epileptic spike is identified to be 41 . Also it is also found from the literature that the total number of samples for epileptic spike is 41 , for a 256 samples per second sampling rate of acquiring the EEG $[1,44]$.

Hence we have employed a moving window size of 41 samples for ApEn calculation on the EEG signals. This window moves for every sample and calculates the ApEn for the next 41 samples. In essence, this window searches for the epileptic spike. Till the occurrence of the epileptic spike, this window gives the ApEn values for the normal EEG signal for each sample and the forthcoming 41 samples. These ApEn values will be generally larger. But at the onset of epileptic seizure, the ApEn value drops due to some regularity nature of the epileptic seizures. This dropping of ApEn value at onset of epileptic seizures is shown in the Figure 3, where we can see that at the sample 4048 where the epileptic seizure is getting onset, the ApEn value goes to 0.6671 . Thus this puts the signal in the ApEn domain forming our feature for automatically detecting the onset of epileptic seizures.

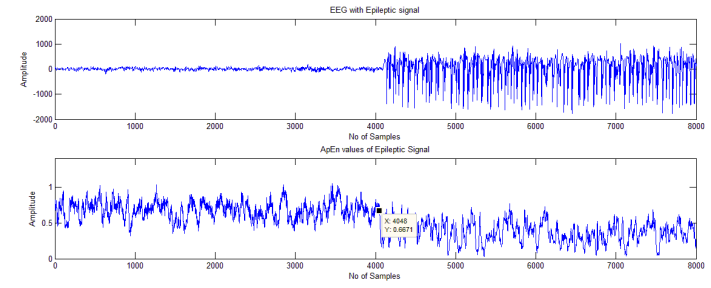

Figure 3. ApEn drop at the onset of epileptic seizure.

\section{Wavelet transform}

In order to enhance the characteristic drop in the ApEn domain and therefore to have a clear discrimination at the onset of epileptic seizure, we applied discrete wavelet transform to this signal. In order to choose a wavelet basis function that matches the shape of ApEn characteristic drop, we computed correlation between the shape of ApEn characteristic drop and various wavelet bases available in the Matlab tool box. Based on the maximum correlation obtained we chose Daubechies 2 (DB2) wavelet. Figure 4 shows the similarity between DB2 
wavelet and the shape of the ApEn characteristic drop. This type of approach is not available in the literature as far as the knowledge of the author and thus forming a part of the novelty of this work. We decomposed this signal using Db2 wavelet up to 5 levels. The decomposed signal is shown in Figure 5. The signal is reconstructed using soft threshold method and the reconstructed signal is shown in Figure 6. This is referred as the feature ApEn in wavelet framework.

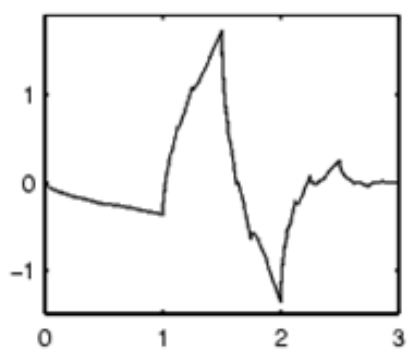

A

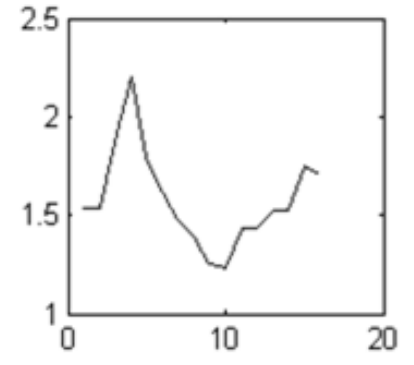

B
Figure 4. A) Db2 wavelet; B) The signal at the ApEn drop at the onset of epileptic seizure.
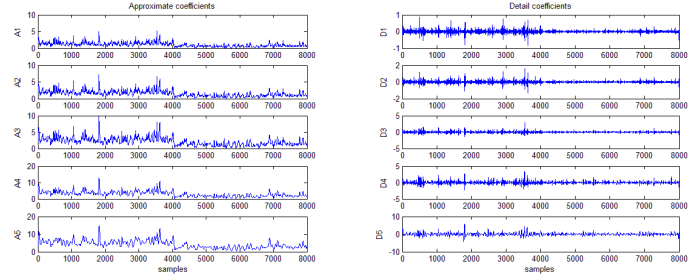

Figure 5. Db2 Wavelet Decomposition of the signal in ApEn domain.

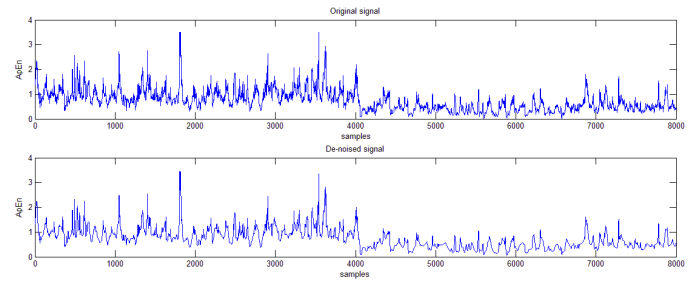

Figure 6. Reconstructed or Denoised Signal of the ApEn domain using wavelet transform.

\section{Neural network classifier}

The reconstructed signals are to be used as inputs to the neural networks to automatically identify the onset of the epileptic seizures. Artificial neural networks (ANN) are proven to be good classifiers due to their inherent characteristics such as self organization, adaptive learning, and robustness. They are useful in situations where enough data are available for training. Different types of ANNs have been used for the detection of epileptic seizures in the literature [43-47]. However here we are using the feature ApEn in wavelet framework as input to the ANN. Here we have employed three different neural networks namely feed forward back propagation network, radial basis network, Elman network are tried to automatically detect the onset of epileptic seizures.
Salient details of the three neural networks, about its target, configuration and threshold values are given below.

Feed forward network: The feed forward algorithm is simple and the information moves only in one direction and there are no cycles or loops. For the training of the network we use back propagation algorithm in which the error in the data generated is propagated back into the layer by using appropriate weights and the threshold values (Bias). The feed forward neural network we used consists of one hidden layer with 5 nodes (neurons in the hidden layer) and the transfer function used for classification is tan-sigmoidal function in hidden layer as well as in the output layer. The output layer has only one node. The gradient descent algorithm is used for back propagation training of the neural network. The input to the neural network is the feature ApEn in wavelet framework. In the neural network, the target is fixed as follows: Epileptic signal is given a value of 0 and for non-epileptic signal the value is 1 . The neural network classify the epileptic signal within a range of values 0 to 0.3 and non-epileptic from 0.8 to 1 .

Radial basis network: The radial basis network uses interpolation technique as tool to perform classification. The activation function used is Gaussian radial basis function. The radial basis network adds the neurons to the hidden layer to reach the specified mean squared error. The network consists of an input layer, a hidden layer and an output layer. We are assuming the maximum number of neurons that can be added to the layer is 25 and the number of neurons at each stage of the iteration to be added is 1 . The input to network is similar to the feed forward network. The target to the neural network is fixed as: epileptic signal is 0 and non- epileptic signal is 1 . After classification the values generated by the neural network for epileptic signal is within the range of 0 to 0.4 and for nonepileptic signal are 0.7 to 1.07 .

Elman network: Elman network is a type of recurrent layer network with two hidden layers. In Elman network it has a special copy layer where it stores the previous pattern and used for the communication between the hidden layers. Thus the network works with the current input values, past records of states of the neurons and the output values. For training of the network we use back propagation algorithm in which the error is propagated through both the hidden layers to the input layer. The numbers of nodes used in the hidden layers are 90. The transfer function used in the hidden layer for classification is tan-sigmoidal and in the output layer is log-sigmoidal. The output layer has one node. The gradient descent algorithm is used for training of the network as a tool for back propagation of the error by using suitable weights and threshold values. The target to the neural network is fixed as: epileptic signal is 1 and non- epileptic signal is 0 . The classified values generated by the neural network for the epileptic signal is above 0.26 and for the non-epileptic signal it is below 0.2. For all the neural networks, we have used 125 data for training and the remaining 75 for testing. 


\section{Performance evaluation parameters}

The performance of Feed forward Back propagation network, Radial basis network and Elman network are evaluated by using three parameters, namely, sensitivity(SE), specificity (SP), and over all accuracy (OA) [1].

\section{$\mathrm{SE}(\%)=(\mathrm{TNcp} / \mathrm{TNap}) * 100$}

Where TNcp represents the total number of correctly detected positive patterns and TNap represents the total number of actual positive patterns. A positive pattern indicates a detected seizure.

\section{$\mathrm{SP}(\%)=(\mathrm{TNcn} / \mathrm{TNan}) * 100$}

Where TNen represents the total number of correctly detected negative patterns and TNan represents the total number of actual negative patterns. A negative pattern indicates a detected non-seizure.

$\mathrm{OA}(\%)=(\mathrm{TNcdp} / \mathrm{TNapp}) * 100$

Where TNcdp represents the total number of correctly detected patterns and TNapp represents the total number of applied patterns, a pattern indicates both seizure and non-seizure.

\section{Results}

The above method was applied to 200 data sets. At first we have trained and tested the three different neural network by using the ApEn values obtained from the data without using wavelet transform for enhancing the characteristic drop of ApEn or denoising them. The output of the neural networks namely Radial basis network, Elman network and Feed forward back propagation network are shown in the Figures 7-9 respectively. From Figure 9 it can be seen that there are a number of places where the feed forward back propagation network wrongly identified the seizure onset. From Figure 8 it can be found that the Elman network has very narrow discrimination ( 0.217 for normal and 0.23 for epileptic seizure) for classifying the onset of epileptic seizures. From Figure 7 it is observed that the Radial basis network is able to clearly discriminate the onset of epileptic seizure inspite of the presence of noise in the data in ApEn domain.

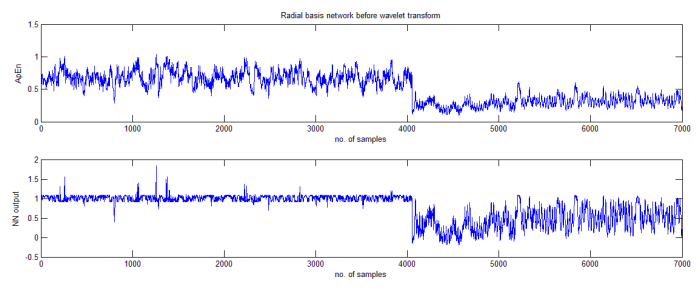

Figure 7. Radial basis network before wavelet denoising.

Secondly we have also trained and tested the three different neural network by using the feature ApEn in wavelet framework. The output of the neural networks namely Radial basis network, Elman network and Feed forward back propagation are shown in the Figure 10-12 respectively. From Figure 11 it can be seen that Elman network doesnot show any significant improvement in discriminating the onset of epileptic seizures even after enhancing the ApEn characteristic drop using wavelet transform ( 0.2 for normal and 0.25 for epileptic seizure). From Figure 10 it is found that the Radial basis network did not respond to transients in the signal and also detected the onset of the epileptic seizure accurately, in comparison with itself (with its input being only ApEn feature i.e., before enhancing the characteristic drop) (Figure 7). From Figure 12 we can observe that the feed forward back propagation network performed well in comparison with its own (with its input being only ApEn feature i.e., before enhancing the characteristic drop) (Figure 9). It is seen that this network was not only able to detect the onset of epileptic seizure accurately but also it was able to detect the termination of the epileptic seizure. It is also inferred that after denoising the input or enhacing the characteristic drop and giving that as feature (ApEn in wavelet framework) for this network it did not respond to the transients. On the other hand we can also observe that the Radial basis network was only able to detect the onset accurately and not the termination of the epileptic seizure. All the figures shown has the same number of segments as the input for training for better visual comparison and only for radial basis network only one segment is shown for visual clarity of the network output at the onset of epileptic seizure.

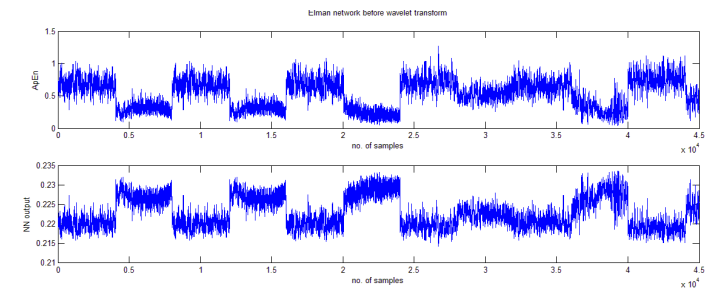

Figure 8. Elman Network before wavelet denoising.

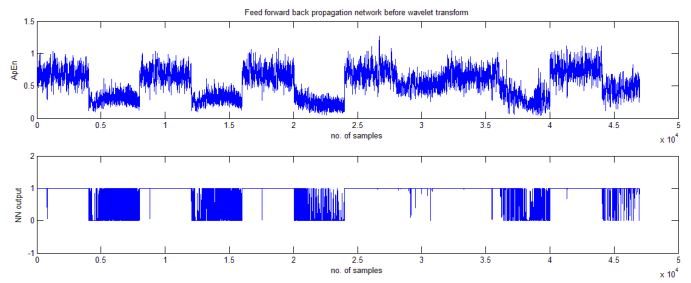

Figure 9. Feed forward Back propagation Network before wavelet transform.

On the whole we find that feed forward network is only able to discriminate the onset as well as the termination of the epileptic seizure with its input as feature: ApEn in wavelet framework. Elman network doesnot show fine dicrimination between the normal signal and the epileptic seizure either with its input being only the ApEn feature or ApEn in wavelet framework feature to the network. Another disadvantage we notice is that eventhough we used 90 nodes for Elman network (which increases the computing time greatly), there is no improvement in the results where as in other neural networks we observe that even with less number of nodes we achieve the required accuracy in the results. Radial basis network was accurately able to detect the onset of the epileptic seizure and 
had only little but important improvement in clarity of the output after its input being feature of ApEn in wavelet framework. It was also inferred that all the networks failed to identify the onset of the epileptic seizure around the sample number 27000 which can be seen in the Figures 7-12, which shows our algorithm had missed onset of the epileptic seizure.

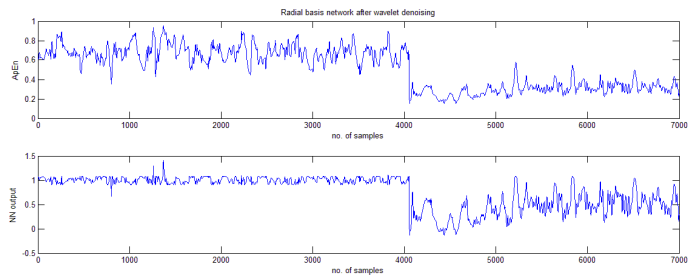

Figure 10. Radial basis Network after wavelet denoising.

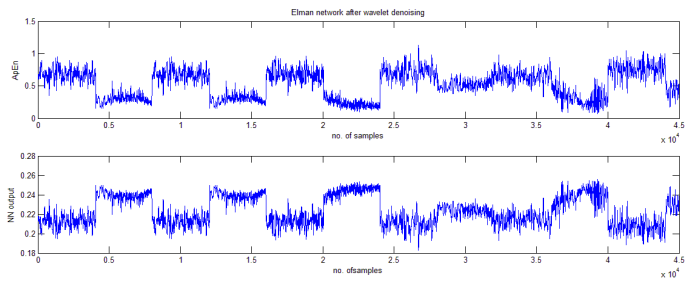

Figure 11. Elman Network after wavelet denoising.

The performance evaluvation for all these networks with ApEn only as feature as inputs and ApEn in wavelet framwork as feature as the inputs is shown in Table 1. Due to the very poor results of the Elman network, we have not listed its performance evaluation in the Table 1. From this table, we can see that radial basis network has better sensitivity, specificity and overall accuracy than other networks and this network also performs well compared to itself after enhancing the ApEn characteritisc drop at the onset of epileptic seizures in the EEG signal through wavelet transform. In the case of feed forward network, the specificity is less due to the occurrence of false positive detection i.e., non epileptic signal being detected as an epileptic signal. Due to this the overall accuracy has also reduced. And we find significant improvement after using the denoised ApEn values using wavelet transform as inputs to the network.

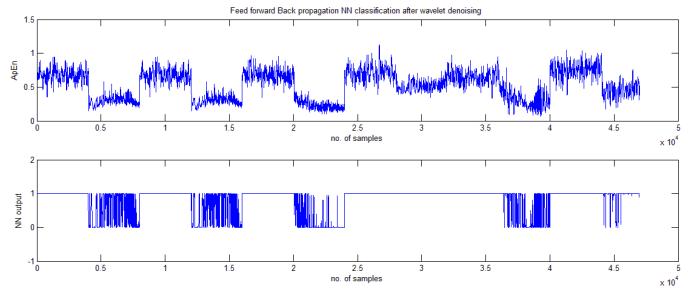

Figure 12. Feed forward Back propagation Network after wavelet denoising.

In addition to the above, we also located the exact onset sample as given by the radial basis network for all the tested 75 data and it was compared with the onset sample point as identified manually by two encephalograpers. Table 2 shows this information for 25 data. The difference in the number of samples between the manually identified sample point by the encephalogrpers and the sample point identified by our algorithm is also shown in the Table 2. The average of this difference is found to be 52 samples, which means that our algorithm was able to detect the onset of epileptic seizures immediately 52 samples which accounts to be only 0.2 second average delay time.

Table 1. Performance Evaluation of the Neural Networks.

\begin{tabular}{|c|c|c|c|c|c|}
\hline \multirow[b]{2}{*}{ Neural Network } & \multicolumn{2}{|c|}{ Before Wavelet Denoising } & \multicolumn{3}{|c|}{ After Wavelet Denoising } \\
\hline & Feed forward back propagation network & Radial basis network & $\begin{array}{l}\text { Feed forward } \\
\text { network }\end{array}$ & back propagation & Radial basis network \\
\hline Sensitivity (SE\%) & 83.5 & 92.5 & 94.5 & & 95.5 \\
\hline Specificity (SP\%) & 50 & 84 & 89 & & 92.5 \\
\hline Over All Accuracy (OA\%) & 62.5 & 87.5 & 91.5 & & 94 \\
\hline
\end{tabular}

Table 2. Epileptic onset detection.

\begin{tabular}{llll}
\hline Data No. & $\begin{array}{l}\text { Visual Epileptic Onset Sample } \\
\text { (By Expert Doctor) }\end{array}$ & Epileptic Onset Sample Detected by our algorithm & Difference in Number of Samples \\
\hline 1 & 4024 & 4076 & 52 \\
\hline 2 & 4032 & 4084 & 52 \\
\hline 3 & 4040 & 4084 & 54 \\
\hline 4 & 4030 & 4100 & 52 \\
\hline 6 & 4048 & 4089 & 52 \\
\hline
\end{tabular}




\begin{tabular}{|c|c|c|c|}
\hline 7 & 4043 & 4095 & 52 \\
\hline 8 & 4023 & 4075 & 52 \\
\hline 9 & 4042 & 4094 & 52 \\
\hline 10 & 4027 & 4080 & 53 \\
\hline 11 & 4025 & 4077 & 52 \\
\hline 12 & 4033 & 4084 & 51 \\
\hline 13 & 4044 & 4096 & 52 \\
\hline 14 & 4029 & 4081 & 52 \\
\hline 15 & 4038 & 4090 & 52 \\
\hline 16 & 4032 & 4084 & 52 \\
\hline 17 & 4047 & 4100 & 53 \\
\hline 18 & 4020 & 4072 & 52 \\
\hline 19 & 4039 & 4091 & 52 \\
\hline 20 & 4045 & 4097 & 52 \\
\hline 21 & 4028 & 4080 & 52 \\
\hline 22 & 4032 & 4083 & 51 \\
\hline 23 & 4037 & 4089 & 52 \\
\hline 24 & 4045 & 4097 & 52 \\
\hline 25 & 4000 & 4052 & 52 \\
\hline
\end{tabular}

Table 3. Comparison of our work with other works.

\begin{tabular}{|c|c|c|c|c|}
\hline Authors & Characteristics & Type of analysis & Sensitivity (\%) & Detection delay \\
\hline Qu and Gotman & Time and frequency domain & Nearest neighbour classifier & 100 & $9.35 \mathrm{~s}$ \\
\hline Shoeb et al. & Wavelet domain & SVM & 94 & $873.2 \mathrm{~s}$ \\
\hline Chan et al & Wavelet domain & SVM & 89.3 & $<3 \mathrm{~s}$ \\
\hline Gabor & Frequency domain & Self-organizing Neural network & 83.8 & l \\
\hline D'Alessandro et al. & $\begin{array}{l}\text { Time, frequency, wavelet-domain and } \\
\text { fractal dimension }\end{array}$ & Probabilistic neural network & 62.5 & l \\
\hline Y. Zhang et al & Wavelet domain & Incremental NDR & 98.8 & $10.8 \pm 5.5 \mathrm{~s}$ \\
\hline Current Study & ApEn in Wavelet Framework & Neural Network (Feed forward and Radial basis) & 95.5 & $0.25 \mathrm{~s}$ \\
\hline
\end{tabular}

\section{Discussion}

There are many seizure onset detection methods which have been developed with different levels of success. For comparison of other works, we have considered the works done in relation to the automatic identification of the onset of the epileptic seizures alone. We have not compared the works done using Bonn University database, as this data has only individual segments of EEG in normal, Ictal and Inter Ictal forms and not an ongoing EEG where the seizure gets onset. $\mathrm{Qu}$ and Gotman implemented KNN classifier. They classified time domain features and frequency domain features independently from each channels [27]. They obtained an average detection delay of $9.35 \mathrm{~s}$ with $100 \%$ onset detection rate. D'Alessandro and Esteller et al. [48] used probabilistic neural network classifier with feature vectors combined along with time, frequency, fractal dimension, wavelet domain-which was used for seizure prediction. The correct prediction rate was only $62.5 \%$. Dastidar et al. introduced a principle component analysis (PCA) based feature enhancement method. PCA was used as feature space from the data which was then given to the radial basis function neural network for classification. This method resulted in an accuracy of $99.3 \%$ [49]. But in this only normal and interictal EEGs was only considered. In our method, we are not using any dimensionality reduction method for feature space creation, rather we are using a moving window ApEn which itself forms as a feature-making it easier 
for real time detection due to its very less computation time. On the other hand, Gabor and Leach implemented a method based on self-organizing neural network which classifies features extracted by two dimensional FFT [18]. This method yielded a sensitivity of $83.8 \%$ only.

Even ApEn was used by Hasan ocak [50], but it was for classifying a standard EEG data segment into say epileptic or normal. He decomposed the EEG signals into wavelet coefficients and calculated the ApEn for these coefficients. He achieved a classification rate of $96 \%$ for the Bonn university data which are noise free data segments already categorized into seizure and normal. Here we are using ApEn for identifying the onset of epileptic seizure in a raw EEG and specifically the ApEn characteristic drop is further enhanced with a specific wavelet transform. Our work although uses ApEn, but it is for a completely different purpose. The work of Hasan ocak is discussed just to bring to the light that ApEn has been used in epilepsy detection. Similarly Srinivasan et al. [43] used ApEn over selected segments of EEG data each with 23 second duration. Again he did not use it for the onset detection, rather he analyzed the various parameters of ApEn for proper classification of the EEG segment considered to be either epileptic or normal. Similar work was also done by Guo et al. [51], where ApEn features are derived from the wavelet coefficients of the data from Bonn University. But in our work, we have used the ApEn characteristic drop enhanced by wavelet transform as a feature for the onset detection as well as the detection delay, which the above authors have not done. Shoeb and Edwards used discrete wavelet transform to construct spectral features in all channels of EEG and used SVM for classification [25]. This method obtained $94 \%$ sensitivity with an average delay of 8 seconds. In our method, we have used wavelet transform in the ApEn feature domain. Kharbouch et al. build on Shoeb method and achieved a sensitivity of $97 \%$ and could reduce the average delay to only 3.5 seconds [52]. Although the overall accuracy obtained in our work is $94 \%$ the average detection delay is only 0.2 second which is very low compared to other works in the literature.

The novelty of our work is discussed as follows. Although researchers have used ApEn as a feature for detecting the EEG components like ictal, normal and interictal [14], there is no specific work in relation to identifying the onset of epileptic seizures using ApEn in wavelet framework as far as the knowledge of the authors. Here we have enhanced the characteristic drop of the ApEn at the conjuncture of seizure onset using a specific wavelet which matches the characteristic drop of the ApEn. This puts the ApEn feature in wavelet framework which we call it as feature in ApEn wavelet framework. Using this as a feature has improved the accuracy and early detection of the onset of the epileptic seizures. We achieved an overall accuracy of $94 \%$ and an average detection delay of only 0.2 second. Moreover the computation time is very less for calculating ApEn, being an important parameter towards building the method for real time onset detection of epileptic seizure. Table 3 shows the comparison of our work with others in the literature. It is found that our method has got the least average detection delay of 0.2 second when compared to other methods. The limitations of our work can be seen as follows. In terms of overall accuracy and sensitivity, we got $94 \%$ and $95.5 \%$ using radial basis network respectively. This is relatively lower compared to the method developed by Qu and Gotman [27] and Zhang et al. [53] who achieved a sensitivity of $100 \%$ and $98.8 \%$ respectively. Also we have manually extracted 200 segments of artifact free EEG data of standard fixed length across 31 patients, wherever the seizure is onset as identified by two encephalograpers. Hence this is not the running long term EEG for hours where our method was tested. Also we have considered only artifact free EEG data as input to our method which is not in general, the practical case. Moreover ApEn depends on the sample length, thereby limiting its use towards a fixed sample length of EEG data for processing. With all the above things, we are in the process of working out methods to remove the artifacts automatically as a first stage towards building a fully automated system for real time detection. As mentioned by Acarya et al. [6], the method is planned to be tested on a wide range of data collected at different places so that the method can be applied directly to the any raw EEG data acquired, thereby attempting towards for a fully automated real time onset detection system.

\section{Conclusion and Future Scope}

We have used a moving window ApEn on the segments of EEG data considered. The ApEn value goes down at the onset of the seizure. This characteristic drop of ApEn value is further enhanced using a wavelet transform which puts the feature in ApEn wavelet framework. This is given as inputs to the neural network for classifying. Among the three neural networks tested, it was identified that the radial basis network is identifying the onset of epileptic seizure with an overall accuracy of $94 \%$ and only with an average delay of 52 samples which is only 0.2 second. This average time delay of 0.2 second is less compared to other methods in the literature. As the computation time for ApEn is very small, we are in the process of working out methods to remove the artifacts automatically and thereby to test the method on a wide range of data collected at different places so that the method can be applied directly to the raw EEG data, thereby attempting for a fully automated system.

\section{References}

1. Indiradevi KP, Elias E, Sathidevi PS, Nayak SD, Radhakrishnan K. A multi-level wavelet approach for automatic detection of epileptic spikes in the electroencephalogram. Comput Biol Med 2008; 38: 805-816.

2. Acharya UR, Molinarib F, Sreec SV, Chattopadhyayd S, Nge KH, Suri JS. Automated diagnosis of epileptic EEG using entropies. Biomed Signal Process Control 2012.

3. Nicolaou N, Georgiou J. Detection of epileptic electroencephalogram based on Permutation Entropy and Support Vector Machines. Expert Syst Appl 2012. 
4. Acharya UR, Sree SV, Suri JS. Use of principal component analysis for automatic detection of epileptic EEG activities. Expert Syst Appl 2012; 39: 9072-9078.

5. Callaway E, Harris PR. Coupling between cortical potentials from different areas. Science 1974; 183: 873-875.

6. Acharya UR. Automated EEG analysis of epilepsy: A review. Knowl Based Syst 2013.

7. Murro AM, King DW, Smith JR, Gallagher BB, Flanigin HF, Meador K. Computerized seizure detection of complex partial seizures Electroenceph. Clin Neurophysiol 1991; 79: 330-333.

8. Harding GW. An automated seizure monitoring system for patients with indwelling recording electrodes Electroenceph. Clin Neurophysiol 1993; 86: 428-437.

9. Yoo CS, Jung DC, Ahn YM, Kim YS, Kim SG, Yoon H, Lim YJ, Yi SH. Automatic detection of seizure termination during electroconvulsive therapy using sample entropy of the electroencephalogram. Psychiatry Res 2012; 2012: 76-82.

10. Majumdar K. Differential operator in seizure detection. Comput Biol Med 2012; 42: 70-74.

11. Acharya UR, Vinitha Sree S, Chattopadhyay S, Wenwei YU, Alvin APC. Application of recurrence quantification analysis for the automated identification of epileptic EEG signals. Int J Neural Syst 2011; 21: 199-211.

12. Acharya UR, Sree SV, Suri JS. Automatic detection of epileptic EEG signals using higher order cumulant features. Int J Neural Syst 2011; 21: 403-414.

13. Acharya UR, Sree SV, Alvin AP, Yanti R, Suri JS. Application of non-linear and wavelet based features for the automated identification of epileptic EEG signals. Int $\mathrm{J}$ Neural Syst 2012; 22: 1250002.

14. Acharya UR, Molinari F, Vinitha Sree S, Chattopadhyay S, $\mathrm{Ng} \mathrm{KH}$, Suri JS. Automated diagnosis of epileptic EEG using entropies. Biomed Signal Process Control 2012; 7 : 401-408.

15. Sartoretto F, Ermani M. Automatic detection of epileptiform activity by single-level wavelet analysis. Clin Neurophysiol 1999; 110: 239-249.

16. Subashi A. Automatic detection of epileptic seizure using dynamic fuzzy neural networks. Expert Syst Appl 2006; 31 : 320-328.

17. Sharma A, Wilson SE, Roy R. EEG classification for estimating anesthetic depth during halothane anesthesia. In:proc, 14th annual int.coof. IEEE engineering in medicine and biology society.IEEE,Newyork, 1992.

18. Gabor AJ, Leach RR, Dowla FU. Automated seizure detection using a self-organizing neural network, Electroencephalography. Clin Neurophysiol 1996; 99: 257-266.

19. Ubeyli ED, Guler I. Features extracted by eigenvector methods for detecting variability of EEG signals. Pattern Recog Lett 2007; 28: 592-603.

20. Ghosh-Dastidar S, Adeli H. Improved spiking neural networks for EEG classification and epilepsy and seizure detection. Integrated Computer-Aided Eng 2007; 14: 187-212.

21. Ghosh-Dastidar S, Adeli H. Spiking neural networks. Int J Neural Syst 2009; 19: 295-308.

22. Ghosh-Dastidar S, Adeli H. A new supervised learning algorithm for multiple spiking neural networks with application in epilepsy and seizure detection. Neural Networks 2009; 22: 1419-1431.

23. Postnov DE, Ryazanova LS, Zhirin RA, Mosekilde E, Sosnovtseva OV. Noise controlled synchronization in potassium coupled neural networks. Int J Neural Syst 2007; 17: 105-113.

24. Chen M, Jiang CS, Wu QX, Chen WH. Synchronization in arrays of uncertain delay neural networks by decentralized feedback control. Int J Neural Syst 2007; 17: 115-122.

25. Shoeb A, Edwards H, Connolly J, Bourgeois B, Treves ST, Guttag J. Patient- specific seizure onset detection. Epilepsy Behav 2004; 5: 483-498.

26. Guler E, Ubeyli D. Multiclass support vector machines for EEG signals classification. IEEE Transact Informa Technol Biomed 2007; 11: 117-126.

27. Qu H, Gotman J. A patient-specific algorithm for the detection of seizure onset in long-term EEG monitoring: possible use as a warning device. IEEE Transact Biomed Eng 1997; 44: 115-122.

28. Saab ME, Gotman J. A system to detect the onset of epileptic seizures in scalp EEG. Clin Neurophysiol 2005; 116: 427-442.

29. Elger CE, Lehnertz K. Seizure prediction by non-linear time series analysis of brain electrical activity. Euro J Neurosci 1998; 10: 786-789.

30. Martinerie J, Adam C, Le Van Quyen M, Baulac M, Clemenceau S. Epileptic seizures can be anticipated by non-linear analysis. Nat Med 1998; 4: 1173-1176.

31. Iasemidis LD. Phase space topography and the Lyapunov exponent of electrocorticograms in partial seizures. Brain Topography 1990; 2: 187-201.

32. Van Drongelen W, Nayak S, Frim DM. Seizure anticipation in pediatric epilepsy: use of Kolmogorov entropy. Pediatric Neurol 2003; 29: 207-213.

33. Khan Y, Gotman J. Wavelet based automatic seizure detection in intracerebral electroencephalogram. Clin Neurophysiol 2003; 114: 898-908.

34. Guler E, Ubeyli D. Adaptive neuro-fuzzy inference system for classification of EEG signals using wavelet coefficients. J Neurosci Methods 2005; 148: 113-121.

35. Hopfengartner R. An efficient, robust and fast method for the offline detection of epileptic seizures in long-term scalp EEG recordings. Clin Neurophysiol 2007; 118: 2332-2343.

36. Adeli H, Ghosh-Dastidar S, Dadmehr N. A wavelet-chaos methodology for analysis of EEGs and EEG sub-bands to detect seizure and epilepsy. IEEE Transact Biomed Eng 2007; 54: 205-211.

37. Ghosh-Dastidar S, Adeli H, Dadmehr N. Mixed-band wavelet-chaos-neural network methodology for epilepsy 
and epileptic seizure detection. IEEE Transact Biomed Eng 2007; 54: 1545-1551.

38. Adeli H, Ghosh-Dastidar S, Dadmehr N. A spatio-temporal wavelet-chaos methodology for EEG-based diagnosis of Alzheimer's disease. Neurosci Lett 2008; 444: 190-194.

39. Pincus S. Approximate entropy (ApEn) as a complexity measure. Chaos 1995; 5: 110-117.

40. Arunkumar N, Sirajudeen M. Approximate Entropy Based Ayurvedic Pulse Diagnosis for Diabetics - A Case Study, IEEE.

41. Arunkumar N, Jayalalitha S, Venugopal A, Dinesh S, Sekar D. Sample Entropy based Ayurvedic Pulse Diagnosis for Diabetics. IEEE Int Conference Advances Eng Sci Manag 2012; 2: 65-66.

42. Arunkumar N, Jayalalitha S, Venugopal A, Dinesh S, Sekar D. Automatic Identification of Acute Arthristis from Ayurvedic Wrist Pulses. IEEE Int Confer Elect Comput Technol 2012; 1: 506-508.

43. Srinivasan V, Eswaran C, Sriraam N. Approximate Entropy - based Epileptic EEG detection using Artificial Neural Network. IEEE Transact Informa Technol Biomed 2007; 11: 3 .

44. Arunkumar N, Balaji VS, Subhasree V, Lihitha R, Sivakama SS. Automatic detection of epileptic seizures using independent component analysis. IEEE International Conference on Advances in Engineering, Science and Management 2016.

45. Wilson SB. A Neural Network method for automatic and incremental learning applied to patient-dependent seizure detection. Clin Neurophysiol 2005; 116: 1785-1795.

46. Naghsh-Nilchi AR, Aghashahi M. Epilepsy seizure detection using eigen-system spectral estimation and Multiple layer perceptron neural network. Biomed Signal Process Control 2010; 5: 147-157.
47. Kumar SP, Sriraam N, Benakop PG, Jinaga BC. Entropies based detection of epileptic seizures with artificial neural network classifiers. Expert Syst Appl 2010; 37: 3284-3291.

48. D’Alessandro M, Esteller R, Vachtsevanos G. Epileptic seizure prediction using hybrid feature selection over multiple intracranial EEG electrode contacts: a report of four patients. IEEE Transact Biomed Eng 2003; 50; 603-615.

49. Ghosh-Dastidar S, Adeli H, Dadmehr N. Principal component analysis-enhanced cosine radial basis function neural network for robust epilepsy and seizure detection. IEEE Transact Biomed Eng 2008; 55: 512-518.

50. Ocak H. Automatic detection of epileptic seizures in EEG using discrete wavelet transform and approximate entropy. Expert Syst Appl 2009; 36: 2027-2036.

51. Guo L, Rivero D, Pazos A. Epileptic seizure detection using multiwavelet transform based approximate entropy and artificial neural networks. J Neurosci Methods 2010; 193: 156-163.

52. Kharbouch A, Shoeb A, Guttag J, Cash SS. An algorithm for seizure onset detection using intracranial EEG. Epilepsy Behav 2011; 22 Suppl 1: S29-35.

53. Zhang Y, Xu G, Wang J, Liang L. An automatic patientspecific seizure onset detection method in intracranial EEG based on incremental nonlinear dimensionality reduction. Comput Biol Med 2010; 40: 889-899.

\section{*Correspondence to}

N. Arunkumar

Department of Electronics and Instrumentation Engineering

Sastra University

India 